This is the accepted manuscript of the article, which has been published in International

Journal of Human Resource Management. http://dx.doi.org/10.1080/09585192.2017.1396551

PERSONAL SOCIAL MEDIA USE AT WORK

\title{
Staying in touch while at work: Relationships between personal social media use at work and work-nonwork balance and creativity
}

This manuscript has been accepted for publication in The International Journal of Human

\author{
Resource Management \\ Accepted version
}

September, 2017

Jana Kühnel

Department of Work and Organizational Psychology, Ulm University, Ulm, Germany

Department of Work and Organizational Psychology, University of Mannheim, Mannheim, Germany

\section{Tim Vahle-Hinz}

Department of Occupational Health Psychology, Humboldt University of Berlin, Berlin, Germany

Jessica de Bloom

Department of Psychology, Faculty of Social Sciences, University of Tampere, Tampere, Finland

Faculty of Economics \& Business, University of Groningen, Groningen, Netherlands

Christine J. Syrek

Department of Work and Organizational Psychology, University of Trier, Trier, Germany 
PERSONAL SOCIAL MEDIA USE AT WORK

Correspondence to: Jana Kühnel, Department of Work and Organizational Psychology, Ulm University, 89069 Ulm, Germany. E-mail: jana.kuehnel@uni-ulm.de

Acknowledgements: We thank our students Nadine Bruhn, Julia Dietz, Ia Enukidze, Kirsten Dorothea Fey, Viola Geißel, Nora Kischhöfer, Annette Knoblauch, Gregor Matheis, and Finja Sonntag for assisting us in collecting the data for this study. 


\title{
Staying in touch while at work: Relationships between personal social media use at work and work-nonwork balance and creativity
}

\begin{abstract}
Personal social media use at work is usually deemed counterproductive work behaviour reducing employee productivity. However, we hypothesized that it may actually help employees to coordinate work and nonwork demands, which should in turn increase work-related creativity. We used ecological momentary assessment across one working day with up to ten hourly measurements on 337 white-collar workers to measure personal social media use, work-nonwork balance and creativity, resulting in a total of 2,244 hourly measurements. Multilevel modelling revealed that personal social media use was associated with better work-nonwork balance, but with lower levels of creativity between- and within-persons. Work-nonwork balance did not mediate the relationship between personal social media use and creativity. More research is needed to understand why employees use social media at work for personal purposes and how this affects their well-being and job performance.
\end{abstract}

Keywords: social media use, work-home balance, work-family balance, creativity, ecological momentary assessment, micro-break

Social media use has dramatically increased within the last decade. It refers to "a group of Internet-based applications that build on the ideological and technological foundations of Web 2.0, and that allow the creation and exchange of user generated content" (Kaplan \& Haenlein, 2010, p. 61). A key element of social media use is that it allows for social presence to emerge between at least two communication partners (Kaplan \& Haenlein, 2010). The majority of American adults use at least one social media device regularly (Duggan, Ellison, Lampe, Lenhart, \& Madden, 2015; Perrin, 2015). More importantly, social media permeates all spheres of life and employees increasingly use social media at work for personal purposes. Surveys suggest that employees may spend up to two hours of their daily working time on personal online activities such as writing personal emails, instant messaging, and social networking (e.g., Henle, Kohut, \& Booth, 2009; Vitak, Crouse, \& LaRose, 2011). The use of social media for personal purposes at work is a new and, according to earlier studies, an 
extremely common behaviour in today's working world. Accordingly, it is important to examine consequences of this behaviour for both organisations and employees alike (Holland \& Bardoel, 2016).

Personal use of social media at work has usually been framed as a counterproductive work behaviour. It may constitute a misuse of working time and company resources, and has therefore often been seen as individual workplace deviance, violating organizational norms (Lim \& Chen, 2012; Robinson \& Bennett, 1995) and impairing individual job performance (Andreassen, Torsheim, \& Pallesen, 2014a; Lavoie \& Pychyl, 2001; Andreassen, Torsheim, \& Pallesen, 2014b). There has so far been very little discussion about the potential beneficial effects of social media use at work (for a discussion about the benefits of non-work media use at work, see Sonnentag \& Pundt, 2017). While previous research suggests that personal social media use at work may be detrimental to job performance, we argue that this negative relationship may not hold true for creativity at work. Moreover, we investigate the potentially beneficial effects of social media use at work by looking at an important, potential outcome neglected so far: employees' work-nonwork balance. More specifically, we propose that personal social media use at work is beneficial for work-nonwork balance, which in turn should foster work-related creativity.

Employees may use social media deliberately to take care of private matters at work, which may reduce role strain and provide them with a feeling of successfully combining work and private life (Olson-Buchanan, Boswell, \& Morgan, 2016). Such a balance of work and nonwork is essential for both employees' well-being and job performance (Allen, Herst, Bruck, \& Sutton, 2000; Beauregard \& Henry, 2009; Kossek \& Ozeki, 1998). A key indicator of job performance is creativity. In modern working life, creativity is essential to help employees cope with and adapt to changes, to solve everyday problems and to come up with innovative ideas to develop and to improve products and processes (Ritter, 2012; Runco, 
2004). Thus, organizational success depends heavily on employees' creativity (Harari, Reaves, \& Viswesvaran, 2016). Personal social media use at work may foster creativity by temporarily distracting employees' attention from a problem at hand. This may give rise to "set shifting", the unconscious recombination of cognitive elements and, as a result, novel ideas and insights (Dijksterhuis \& Meurs, 2006; Sio \& Ormerod, 2009). Personal social media also provides employees with diverse information, which may serve as a source of inspiration. Moreover, employees may transfer successful strategies for creative problemsolving from the private domain to their work and may use analogies derived from their private lives to come up with new ideas at work.

To sum up, we expect to find direct positive links between personal social media use at work, work-nonwork balance and creativity, and we assume that work-nonwork balance is a partial mediator between personal social media use and creativity. If using social media for personal purposes enhances the feeling of having balanced work and personal demands, this should enable employees to refocus attention on work tasks, what in turn should enhance creativity at work.

\section{Research Aims and Design}

The goal of this empirical study is to scrutinize personal social media use to better understand its consequences, challenge the prevailing negative conceptualization for job performance and shed light on the potential benefits of personal social media use at work. In the present study, we conducted an ambulatory assessment study (hourly measurements during one working day) to obtain detailed information on the effects of personal social media use at work on work-nonwork balance and work-related creativity. This approach minimizes recall bias (Bolger, Davis, \& Rafaeli, 2003; Ohly, Sonnentag, Niessen, \& Zapf, 2010), and allows us to investigate the effects of personal social media use at work on different levels of 
analysis: the between- as well as the within-person level (Beal \& Weiss, 2003). A withinperson effect highlights the hour-specific relationship between personal social media use at work and our outcomes. Specifically, we investigate whether a more extensive use of personal social media during one hour, compared to the personal social media use of that employee during other hours of the working day, is related to higher experienced worknonwork balance and creativity at work during this hour. A between-person effect, on the other hand, shows whether the average use of personal social media at work is associated with employee's general level of creativity at work and work-nonwork balance.

Next, we derive our hypotheses on the relationships between personal social media use at work, work-nonwork balance and job-related creativity in more detail. The conceptual model for our hypotheses is shown in Figure 1.

[Figure 1 about here]

\section{Personal Social Media Use at Work and Work-Nonwork Balance}

Balancing the demands of work and nonwork is an important challenge in today's working world (Kubicek \& Tement, 2016; Major \& Germano, 2006). Many dual-earner couples struggle with heavy demands in both spheres of life (Sparks, Faragher, \& Cooper, 2001). We define work-nonwork balance according to Higgins, Duxbury, \& Johnson (2000) as a “perceptual phenomenon characterized by a sense of having achieved a satisfactory resolution of the multiple demands" of work and nonwork domains (p. 19). This balance is dynamic and varies depending on current experiences (Maertz \& Boyar, 2011). Research shows that work demands vary hourly (Tan \& Netessine, 2014) and we assume that nonwork demands also vary throughout the day (e.g., when children are at school vs. back from school), making the balance between work and nonwork a dynamic challenge. In line with Clark (2000), balance might be achieved more easily if nonwork demands are dealt with by 
crossing the border between work and nonwork. Non-work social media use can be regarded as a border-crossing activity. Social media use at work may help employees to better coordinate work and nonwork demands. A body of research shows that conflict between work and nonwork and an individual's various roles is related to lower well-being and job performance (e.g., Amstad, Meier, Fasel, Elfering, \& Semmer, 2011; Carlson, Kacmar, Grzywacz, Tepper, \& Whitten, 2013). While being at work, it is difficult for employees to attend to demands arising in their private lives. Modern communication devices (e.g., smartphones) and social media apps have made it easier for employees to stay in touch with family members and friends - even when at work - and thus facilitate engagement in private matters when being at work (Ivarsson \& Larsson, 2011; Olson-Buchanan et al., 2016).

In the present study, we suggest that social interactions with the help of modern information and communication technology have a positive effect on employees' ability to fulfil private obligations and successfully combine work and private life. For instance, couples may use social media to organize domestic chores which need to be taken care of after work (e.g., grocery shopping, picking up children from day-care). In addition, social media may enable employees to stay in touch with their significant others despite the physical distance between them. For example, an employee may use social media to provide emotional support to a sick friend or parents may check on their children's activities after school without the need to be physically present. Personal social media use at work may thus serve as a way for employees to take care of unfinished or ongoing personal issues outside work and fulfil different roles at the same time (D'Abate, 2005; Syrek \& Antoni, 2014). Accordingly, personal social media use at work can be seen as a facilitator of work-nonwork balance. We expect that this positive relationship between personal social media use at work and work-nonwork balance will be found when looking at a specific hour of the working day as well as when looking on differences between individuals. Being able to use social media 
for personal purposes during one hour may help to address urgent demands arising from one's private life and should therefore be positively related to work-nonwork balance experienced during that hour (within-person effect). Between individuals, employees who in general use social media for personal purposes more extensively should experience better work-nonwork balance than employees who in general use social media for personal purposes less extensively (between-person effect). Accordingly, we hypothesize:

Hypothesis 1a: During those hours of the working day during which employees use social media for personal purposes more extensively - compared to the hours of the working day during which employees use social media for personal purposes less extensively employees achieve a better work-nonwork balance (within-person effect).

Hypothesis 1b: Employees using personal social media more extensively during working hours achieve a better work-nonwork balance than do those using personal social media less extensively (between-person effect).

\section{Personal Social Media Use at Work and Creativity}

Besides fostering work-nonwork balance, we propose that personal social media use at work should be conducive to creativity at work, defined " $[\ldots]$ as the production of novel, useful ideas or problem solutions" related to work (Amabile, Barsade, Mueller, \& Staw, 2005, p. 368). Employees' creativity is considered a key aspect of companies' competitive advantage and drives organizations forward (Lin, 2011; Scott, Leritz, \& Mumford, 2004). Today’s employee's primary task is to solve "non-routine" problems (Newell, Robertson, Scarbrough, \& Swan, 2009). Therefore, job performance is determined by an employee's ability to acquire, share and utilize knowledge in a creative way (Chen \& Huang, 2009; Kelloway \& Barling, 2000). 
The prevailing assumption is that social media use at work constitutes an off-task concern which distracts employees' attention from the work tasks they need to focus on, consequently reducing their productivity (e.g., Andreassen et al., 2014a; Beal, Weiss, Barros, \& MacDermid, 2005). However, tasks requiring creativity are fundamentally different from routine tasks. For routine tasks, the link between time investment and output is quite direct: more input of time means more output. The link between the sheer investment of time and effort and creative output is less straightforward. Creative insights depend on people's ability to avoid fixating on a problem and to integrate new and diverse information (Lubart, 2001). Research suggests that people working in creative industries use a considerable amount of their working time to engage in personal internet use at work (Vitak et al., 2011), because they look for new and diverse information. We assume that a temporary focus on off-task concerns and subsequent redirection of attention to work tasks helps employees to solve problems creatively and to generate creative ideas. Personal social media use "might prevent them from getting stuck in a situation" or fixated on suboptimal problem solutions (Ivarsson \& Larsson, 2011, p. 75) and instigate "set shifting". Set shifting distracts employees from the problem at hand, which they have tried to solve using the wrong cues, faulty or incomplete information or inappropriate heuristics (Dijksterhuis \& Meurs, 2006; Sio \& Ormerod, 2009). After engaging in different activities for a while (e.g., chatting with a family member, organizing home chores, or reading updates of friends on social networks), this biased approach to the problem, or fixated thinking style is less pervasive and the employee may be able to take a "fresh perspective on" the old problem and come up with new strategies and ideas to solve it (Schooler \& Melcher, 1995). In creativity research, this stage in creative problem solving during which the problem solver is not consciously working on the problem has been referred to as "incubation" (Guilford, 1979; Lubart, 2001). 
In addition, social media use may present the employee with interesting new and diverse pieces of information. A crucial element of social media is that the sources of information are manifold (e.g., family members, friends, groups to which a person is connected). Consequently the information which employees receive is diverse. According to Simonton's evolutionary theory of creativity $(1999 ; 2010)$, creative insights depend on two factors: variation (generation of breadth of ideas) and selection (selection of the best idea). Exposure to diverse information increases creativity, because the number of cognitive elements available for association and selection is wider (Amabile et al., 2005; Dijksterhuis \& Meurs, 2006; Kühn et al., 2013).

In addition to "set shifting" and access to diverse information, personal social media use may induce positive affect or may instigate an affective shift from negative to positive affect, both of which are conducive to creativity at work (Bledow, Rosing, \& Frese, 2013; Fredrickson, 2001). Positive activated mood states are conducive to creativity because they broaden the scope of attention (that is, increase the number of cognitive elements available for association) and the scope of cognition (that is, they increase the breadth of those elements that are seen as relevant to the problem; Amabile et al., 2005; Baas, De Dreu, \& Nijstad, 2008; Sio, Monaghan, \& Ormerod, 2013). Consequently, employees should be more likely to come up with creative solutions for problems and to generate creative ideas.

Taken together, during the hours of the working day when employees engage in personal social media use, employees should achieve greater creativity than during the hours of the working day when they engage less in personal social media use. In addition, employees who in general use personal social media at work more extensively should experience greater creativity at work than do employees who generally use personal social media less while at work. We hypothesize: 
Hypothesis 2a: During those hours of the working day during which employees use social media for personal purposes more extensively - compared to the hours of the working day during which employees use social media for personal purposes less extensively employees achieve higher creativity (within-person effect).

Hypothesis 2b: Employees using personal social media more extensively during working hours achieve greater creativity than do those using personal social media less extensively (between-person effect).

\section{Work-Nonwork Balance as a Partial Mediator in the Relationship between Social Media Use and Creativity}

We expect that work-nonwork balance is one of the underlying mechanisms that explains the positive relationship between personal social media use and creativity. One reason is that using social media for personal purposes may enhance the feeling of having balanced work and personal demands, which in turn may enable employees to refocus on work and to come up with creative ideas. In terms of the episodic process model (Beal et al., 2005), an off-task attentional pull is decreased when employees have dealt with personal tasks and the competition between private life and work becomes less prominent. As a consequence, more cognitive resources become available for work-related creative ideas. Further, Clark (2000), building on Whetten and Cameron (1998), argues that, "when ideas and insights used in one situation can be transferred to another, they can be seeds of creativity" (p. 756). It may be that solutions to nonwork-related problems can be translated into solutions to work-related problems. Following Clark (2000), we assume that being able to draw abstract conclusions and using a strategy for work that has proven efficient in personal situations describes how work-nonwork balance may lead to creative work-related ideas. We therefore expect that personal social media use improves work-nonwork balance, which in turn enhances employees' creativity at work. 
Hypothesis 3: There is a positive, indirect relationship between personal social media use at work and creativity via enhanced work-nonwork balance (within-person effect).

\section{Method}

\section{Ambulatory Assessment Approach}

In the present study, we used an ambulatory assessment approach to investigate the relationship between personal social media use at work, work-nonwork balance and creativity. Specifically, we asked participants hourly during one working day about their hour-specific use of social media for personal purposes, work-nonwork balance and creativity. We utilized this design for several reasons: First, the effects of personal social media use at work may not be long-lasting. Therefore, it is important to evaluate this behaviour in terms of momentary effects. Second, administering short questionnaires relatively close in time to the actual behaviour to be measured reduces recall bias (Ohly et al., 2010). Asking employees about their daily use of social media at the end of the working day may be biased because these interactions (for example checking a text message from the spouse) are typically very short and may easily be forgotten and difficult to recall at the end of a busy working day (Junco, 2013; Reis, 2012). Third, hourly measurements enabled us to differentiate between two levels of analysis: the hourly level (within-person level) and the day level of analysis (between-person level). Thus, we are able to investigate whether the personal use of social media during one hour-compared to the employee's use of social media during other hours of that day—was related to hour-specific creativity at work and work-nonwork balance (hour level, within-person effect). Additionally, we were able to investigate whether the average use of social media at work was associated with the general level of creativity at work and work-nonwork balance (day level, between-person effect). 
Moreover, this research design enabled us to capture and test a fundamental notion of the episodic process model of performance (Beal et al., 2005). This model proposes that the working day is composed of different time episodes. It suggests that cognitive and regulatory processes during one episode determine employees' performance of that episode. Our hourly design enabled us to scrutinize a working day divided into temporal units, and to investigate whether social media use during one temporal unit was relevant for the outcomes of that unit.

\section{Procedure and Design}

Three hundred and thirty-seven employees responded to up to ten electronic hourly surveys across one working day (6.69 times on average). Links to the surveys were sent ten minutes before every full hour to the participants' personal email addresses. The maximum number of obtainable measurements was 3,370. Our data set included 2,244 hourly measurements, meaning a completion rate of $67 \%$. On the next day, respondents received a link to an electronic survey eliciting demographic information and information on their specific working hours the previous day.

\section{Sample}

The sample of our study consisted of 337 white-collar employees in Germany. The respondents were recruited by convenience sampling whereby the authors and their students approached their network of family and friends (response rate 78\%). Participants worked in various organizations in different sectors, the largest of which were engineering, IT and finance. Respondents' mean age was 33.8 years $(S D=10.37$, range 18 to 64$)$, and $50 \%$ of the sample was female. Average weekly working time was 40 hours, average duration of employment was 5.7 years and $74 \%$ of the sample had a permanent employment contract. Eighty percent worked full time. In our sample, the mean length of the working day on which the employees answered the hourly surveys was nine hours $(S D=0.84)$. Fifty-six percent 
held master's or higher academic degrees and 33\% held bachelor's (polytechnic) degrees. The majority (62\%) were married or co-habiting and $24 \%$ had at least one child living with them. As an incentive to participate, participants were offered feedback on the results of the study.

\section{Hourly Measures}

\section{Personal Social Media Use at Work}

Personal social media use at work was measured with the question: "Within the last hour, how much time have you spent using the following media for personal purposes: 1) personal use of social networks (e.g., Facebook, MySpace, chat rooms), 2) personal use of instant messaging (e.g., Whatsapp, Threema, Facebook Messenger), 3) personal emails, 4) personal use of VoIP services (e.g., Skype, Facetime), 5) personal use of social games (e.g., Farmville, Words with Friends), 6) other (please specify)?” Participants could report the exact number of minutes they used these different kinds of social media during the last hour. We calculated the sum of minutes reported in these categories. Minutes reported in the "other" category were only taken into account if the activity reported was included in our definition of personal social media use. For example, chatting in a forum for cat owners was considered personal social media use, while reading a newspaper was not.

\section{Work-Nonwork Balance}

To measure the extent to which employees felt that they were able to combine work and nonwork obligations, we used two items based on the Survey Work Home Interaction NijmeGen (Geurts et al., 2005). The items were adapted to refer to the last hour worked. They read: "Within the last hour, I could fulfil private obligations/duties" and "Within the last hour, I could combine personal life with work". Participants responded on a frequency 
scale ranging from 1 (completely disagree) to 7 (completely agree). Item intercorrelations ranged between .65 and .85 across the hours $(M=.79)$.

\section{Creativity}

Work-related creativity was assessed by two items focusing on having innovative ideas and solving work problems creatively ("Within the last hour, I came up with creative solutions to work problems"; "Within the last hour, my head was full of innovative ideas for my work") adapted from George and Zhou (2001). Participants responded on a frequency scale ranging from 1 (completely disagree) to 7 (completely agree). Item intercorrelations ranged between .72 and .84 across the hours $(M=.77)$.

\section{Control Variables}

We followed Spector and Brannick's (2011) call to consider meticulously the role of control variables and to include only those of theoretical interest. Moreover, we followed the recommendations of Becker et al. (2016) to run analyses with and without control variables and inform the reader about the results.

From work-nonwork research we learned that several background variables must be taken into account. More specifically, we assumed that gender, age, marital status, and cohabiting children may affect people's work-nonwork balance as well as the relationship between social media use at work and work-nonwork balance. For women (who usually take upon themselves a greater share of the childcare and household tasks; e.g., Saxbe, Repetti, \& Graesch, 2011), people who are co-habiting with a partner and/or children and who perceive more emotional demands at home, social media use at work may be more salient than for men, people living without a partner and/or children, and people with fewer emotional demands at home. Consequently, we took the role of these variables into account by assessing whether they directly affected work-nonwork balance (in which case they served as person- 
level control variables) and/or whether they acted as cross-level moderators influencing the relationship between hour-specific personal social media use and work-nonwork balance.

Moreover, the initiator of the contact via social media may be of relevance to the relationship between personal social media use at work and work-nonwork balance. Thus, we assessed whether the use of social media was initiated by the employee herself/himself or by others (self- or other-initiated contact). We used a self-developed item to assess whether the contact via social media was self- vs. other-initiated. Referring to the preceding hour we asked "On average, who initiated contact through social media?" with a 7-point scale ranging from $1=$ I always initiated contact to $7=$ The contact was always initiated by others. If an employee herself/himself took the initiative to engage in personal social media use, it is likely that (s)he had time for a brief social interaction at this point in time during the working day. In this case, attending to a personal matter should be beneficial for the perception of worknonwork balance. If, however, an employee is approached by another person and distracted while (s)he is busy working, this could be experienced as an interruption (Baethge, Rigotti, \& Roe, 2015). Being interrupted may be perceived as a conflict between work and nonwork and thus attenuate the proposed positive relationship between personal use of social media and work-nonwork balance. Thus, we explored whether being the initiator of contact is a moderator of the relationship between social media use and work-nonwork balance.

The creativity literature states that age and job autonomy may explain between-person differences in creativity. High autonomy fosters intrinsic motivation, which is conducive to creativity (Collins \& Amabile, 1999), and the literature suggests that younger or middle-aged people are more creative than older people (McCrae, Arenberg, \& Costa, 1987; Simonton, 1997). Because both age and job autonomy can be related to social media use (younger employees make more use of social media than do older employees; high job autonomy allows employees to engage in personal social media use at work), these variables may create 
a spurious between-person relationship between social media use and creativity. We therefore took into account age, squared age and job autonomy when predicting creativity arising from personal social media use. Job autonomy was measured with four items developed by Semmer, Zapf and Dunckel (1999). Cronbach’s Alpha was .81.

\section{Analytic Strategy}

\section{Multilevel Analysis}

We followed Bliese and Ployhart (2002) in estimating multilevel models in R, using the NLME library written by Pinheiro and Bates (2000), and restricted maximum likelihood for estimation. Multilevel modelling techniques were used to account for the non-independence of the data as well as for the systematic, chronological structure of the data (by including time as a predictor). Because we had implemented ten hourly questionnaires across the working day, the variable "time" ranged from one (referring to the hour between eight and nine o'clock, that is, the nine-o'clock survey) to ten (referring to the hour between five and six o'clock, that is, the six-o'clock survey). We tested for autocorrelation and heteroscedasticity before entering the core predictors. ${ }^{1}$

\section{Person (Between) and Hourly (Within) Effect}

Multilevel analyses make it possible to model between-person effects and within-person effects at the same time. We followed Raudenbush and Bryk (2002) and included hour-level predictors (person-mean centred, depicting within-person variance) and their aggregates (grand-mean centred person-means, capturing the between-person effect over the day), so that the effect was broken down into within- and between-person components. 


\section{Indirect Effect}

To assess the indirect effect of social media use on creativity via work-nonwork balance and take account of the multilevel structure of our data we used the $\mathrm{R}$ mediation package, which estimates the indirect effect and provides respective 95\% quasi-Bayesian confidence intervals (Tingley, Yamamoto, Hirose, Keck \& Imai, 2014).

\section{Results}

[Table 1 about here]

Intercorrelations between the study variables are displayed in Table 1. ICC1 was .42 for work-nonwork balance and .43 for creativity, indicating that roughly half of the variance was due to interindividual differences, and that there was also substantial variance within persons across the working day ( $58 \%$ for work-nonwork balance and $57 \%$ for creativity). Therefore, a multilevel approach was warranted. On average, employees used social media for personal purposes for 4.54 minutes per hour (range 0 to 60 minutes). For the whole working day the average time of using social media was 40.86 minutes. Nine people did not use social media at all for personal purposes during working hours. The most extensively used media were in order of importance: 1) instant messaging, 2) social networks and 3) personal emails. The time trend for personal social media use over the working day was quadratic and positive (that is, u-shaped; $\beta=30.43, S E=5.86, t=5.19, p<.001$ ). Visual inspection of the scatterplot showed that personal social media use peaked at the beginning and at the end of the working day.

[Table 2 about here]

\section{Social Media Use Predicting Work-Nonwork Balance}

For work-nonwork balance, gender $(\beta=.45, S E=.15, t=3.02, p<.01)$, age $(\beta=.02, S E=$ 
$.01, t=2.00, p<.05)$, family status $(\beta=.09, S E=.17, t=0.50, p=.62)$, and having children $(\beta=-.04, S E=.20, t=-0.21, p=.84)$ served as person-level control variables (Model 2, Table 2).

Considering the effects within persons (Model 3, Table 2), the results suggested that personal social media use was related to higher levels of work-nonwork balance withinperson during the same hour $(\beta=.09, S E=.01, t=15.33, p<.001)$. Hypothesis 1a was therefore supported, demonstrating that on an hourly level, personal social media use is associated with better work-nonwork balance.

Personal social media use was also positively related to work-nonwork balance between persons $(\beta=.06, S E=.01, t=4.32, p<.001)$ (Model 3, Table 2). This finding supported Hypothesis 1b, showing that people who used more social media throughout the day reported better work-nonwork balance.

[Table 3 about here]

\section{Social Media Use Predicting Creativity}

For creativity, age $(\beta=.01, S E=.01, t=1.53, p=.13)$, age squared $(\beta=.00, S E=.00, t=$ $0.58, p=.56)$ and job autonomy $(\beta=.18, S E=.08, t=2.36, p<.05)$ served as person-level control variables.

Considering the within-person effect (Model 2, Table 3), the results suggested that personal social media use was significantly but negatively related to creativity within persons $(\beta=-.01, S E=.00, t=-2.60, p<.01)$. This finding did not support Hypothesis 2a proposing a positive relationship between personal social media use and creativity during the same hour.

Personal social media use was negatively related to creativity between persons $(\beta=$ $-.03, S E=.01, t=-2.55, p<.05)$. Hypothesis $2 \mathrm{~b}$, proposing a positive relationship between personal social media use and creativity between persons, was therefore not supported. 


\section{Work-Nonwork Balance as a Partial Mediator between Social Media Use and Creativity}

The indirect effect of personal social media use via work-nonwork balance on creativity was not significant $(a b=-.00004,95 \% C I\{-.00003 ; 0.0004\}, p=.87)$. This finding did not support Hypothesis 3. Additionally, as a test of mediation usually requires measurement of the variables to be separated by time (Maxwell, Cole, \& Mitchel, 2011), we tested our mediation hypothesis by calculating the indirect effect using personal social media use and work-nonwork balance the same hour and creativity the subsequent hour. This analysis likewise showed no significant indirect effect $(a b=-.00008,95 \% C I\{-.0003 ; 0.005\}, p=$ $.70)$.

\section{Potential Cross-Level Moderators of the Relationship between Work-Nonwork}

\section{Balance and Personal Social Media Use}

We explored the role of control variables in the within-person relationship between personal social media use at work and work-nonwork balance, that is, we explored whether control variables explain variance in the slope of personal social media use predicting work-nonwork balance. We found no significant cross-level interaction for gender $(\beta=.03, S E=.02, t=$ $1.61, p=.11)$. Age served as a significant cross-level moderator $(\beta=.002, S E=.001, t=$ $2.85, p<.01$ ), indicating that the positive relationship between social media use and worknonwork balance was stronger for older employees than for younger employees. Further, people living in a relationship benefitted more from social media use at work in terms of better work-nonwork balance $(\beta=.04, S E=.02, t=2.46, p<.05)$. Co-habiting with children did not moderate the relationship between personal social media use at work and worknonwork balance.

In addition, being the initiator of the contact (ranging from $1=$ I always initiated contact to $7=$ The contact was always initiated by others) was significantly related to work- 
nonwork balance $(\beta=-.04, S E=.02, t=-2.24, p<.05)$. That is, during the hours during which the contact was initiated by the social media user studied, more work-nonwork balance was experienced compared to those hours during which the contact was initiated by others. However, the interaction between being the initiator of the personal social media contact and personal social media use at work was not significantly related to work-nonwork balance $(\beta=$ $.00, S E=.003, t=1.33, p=.18)$.

\section{Additional Analyses}

The positive effect of personal social media use on creativity may occur with a time lag. Following "a period during which a problem is 'put aside' often due to an impasse in problem solving" (Lubart, 2001, p. 298), a person may suddenly come up with a creative idea. Because these incubation effects may take some time to surface, we additionally investigated whether social media use at work in one working hour is positively related to creativity in the subsequent hour (time +1 ). The results (Model 3 lagged (time +1 ), Table 3 ) show that personal social media use in one hour marginally positively predicted creativity in the subsequent hour $(\beta=.01, S E=.01, t=1.66, p=.09)$.

We ran all analyses with and without control variables. The overall interpretation of the results for the relationships between non-work social media use, work-nonwork balance and creativity did not change when control variables were included.

\section{Discussion}

\section{Main Findings and Theoretical Implications}

Research on personal social media use at work has so far focused primarily on the "dark side" of this behaviour, referring to it with terms such as cyberloafing (Lim \& Chen, 2012), cyberslacking (Lavoie \& Pychyl, 2001), virtual absenteeism (Friedman, 2001), and goldbricking (Lundgren \& Lundgren, 1999). The aim of our study was to challenge the 
prevailing negative conception of personal social media use at work by investigating its relationship to work-nonwork balance and creativity. The results of our study show that personal social media use at work was positively related to work-nonwork balance. Less conflict between work and private matters is related to better personal (e.g., life satisfaction) and organizational outcomes (e.g., intention to turnover; Amstad et al., 2011). Accordingly, behaviours that facilitate work-nonwork balance can be beneficial for employees and employers alike. Our study supports the notion that personal social media use at work should not be deemed solely counterproductive, because its positive relationship to more distal organizational outcomes (e.g., work-nonwork balance) may warrant a differentiated evaluation of this new and common behaviour in today's working world.

König and Caner de la Guardia (2014) hypothesized that personal internet use at work constitutes a border-crossing behaviour between work and nonwork which should be helpful in achieving work-nonwork balance. However, they found no relationship between personal internet use at work and work-nonwork balance. In contrast to their study, our results demonstrated that personal social media use at work was related to better work-nonwork balance. This seemingly contradictory finding can be explained by the different designs used and the operationalization of internet use. Personal internet use as measured in König and Caner de la Guardia (2014) was rather broad and included browsing news websites or online shopping. We think that social media use for personal purposes may better capture the social interactions needed to reconcile different life domains. In line with this view and work/family border theory (Clark, 2000), personal social media use at work may be framed as border crossing behaviour supporting work-nonwork balance and may be qualitatively different from surfing the web for leisure purposes or online shopping.

With regard to the relationship between personal social media use at work and creativity, our results did not support a positive evaluation of personal social media use at 
work. Albeit the effects were small (Delta Pseudo $R^{2}=.01$ and .02, on Level 1 and Level 2, respectively); people using social media more often at work reported less creativity than people using social media less often at work. In addition, during hours characterized by higher social media use, employees reported less creativity. Therefore, with regard to performance outcomes, our results concur with those of earlier research showing that personal social media use at work may be counterproductive (e. g., Lim, 2002; Weatherbee, 2010). Conversely, additional analyses showed that after hours characterized by higher social media use, employees tended to be more creative than after hours characterized by less social media use. However, this lagged effect was rather small (Delta Pseudo $R^{2}=.01$ ), a finding that diminishes the practical relevance of this effect. More research is therefore needed to investigate the relationship between personal social media use at work and performance outcomes. These studies should carefully consider optimal time lags to measure changes in performance across time. Theoretical suggestions on this matter can be found in Dormann and Griffin (2015). An alternative approach to the use of time lags pre-defined by researchers would be to ask participants to segment their work day into behavioural episodes (Beal et al., 2005).

One might speculate that larger lagged effects for social media use on creativity can be found if measures are used that differentiate between the different types of creative problems employees encountered. Research has shown that not all types of creative problems benefit from an incubation period (Sio \& Ormerod, 2009). Incubation effects were found for creative problems requiring a wide search for information. Problems involving achieving some kind of insight to arrive at a unique solution do not always seem to benefit from incubation. Employees may encounter several types of creative problems in their work. Future research might thus employ measures of creativity that differentiate between different types of creative problems to reveal incubation effects due to social media use. 
Contrary to expectations, we could not establish beneficial relationships between social media use and creativity. Theoretically, we assumed several pathways by which personal social media use at work fosters creativity: set shifting, access to diversified information and increasing positive affect. Concerning diversity of information, public discussions have recently focused on social media networks as "echo chambers". Instead of diverse information, users may primarily be confronted with information they are already familiar with and opinions they share with like-minded people (Edwards, 2013). This may limit the potential of social media use to broaden employees' horizons and incorporate radically new ideas from their personal networks into their work. Although social media use seems to enable employees to balance work and nonwork demands, interactions with family members may not necessarily induce positive affect. For instance, if couples argue about domestic tasks via social media or receive bad news from friends, this may actually impair their mood and induce negative affect. In this state of mind, creative ideas may be less likely to come to mind. We thus recommend that future research should try to measure these potential mediators, for example by assessing and evaluating the content of the social media with which employees engage. We do, however, acknowledge that this endeavour poses a major challenge with regard e.g. to protection of privacy and content analysis.

Our study did not support work-nonwork balance as a mediator of the relationship between personal social media use at work and creativity. In our study, work-nonwork balance and creativity were neither significantly related to each other within-persons $(r=-$ $.04, n s)$ nor between-persons $(r=.004, n s)$. Thus, hour-specific work-nonwork balance does not seem to be conducive to hour-specific creativity at work. This finding contradicts the research so far: In a meta-analysis investigating cross-sectional relationships only, Amstad et al. (2011) showed that work-nonwork imbalance is negatively related to work-related performance. Further research is needed, because - to the best of our knowledge - there is no 
other experience sampling study investigating the momentary, that is, hour-specific or dayspecific relationships between work-nonwork balance and creativity. Future studies might benefit from including different performance measures to clarify the potential benefits of momentary work-nonwork balance for short-term fluctuations in performance.

Another fruitful approach for future research could be to apply the framework of the self-determination theory (Ryan \& Deci, 2000) when studying personal social media use at work. It is conceivable that personal social media use helps to satisfy an employee's need for autonomy and relatedness which in turn is expected to foster well-being and performance (e.g., Ryan, Bernstein, \& Brown, 2010).

\section{Limitations and Suggestions for Future Research}

There are several limitations that should be taken into account. Ambulatory momentary (i.e. hourly) assessment can be regarded as a means to assess "life as it is lived", significantly reducing recall bias and improving ecological validity. Nevertheless, employees' hourly selfreports of the duration of social media use might still be inaccurate. There are large interindividual differences concerning time perception as well as contextual influences on duration estimates (for reviews, see Matthews \& Meck, 2014 and Block \& Zakay, 1997). Thus, it would have been preferable to collect additional data assessed through automatic logging or apps monitoring employees' social media use. Yet tracking options and monitoring applications can be regarded as intrusive and profoundly critical in terms of data privacy.

A second limitation is the possibility of employees' reports being biased by social desirability, that is, systematically underreported, because personal social media use at work is typically perceived as an undesirable work behaviour. Comparing our results (personal social media use of 4.54 minutes per working hour, 40.83 minutes per working day) to that 
reported in earlier studies (e.g., Henle et al., 2009; Vitak et al., 2011), we arrive at the assumption that the employees in our study may have used personal social media less than usual. For the within-persons relationships, this is, however, less problematic, because a general tendency to report fewer minutes of social media use at all measurement points does not change the interpretation of our within-persons results. However, it would have been problematic if employees' use of social media during the day had been systematically curtailed over time due to the repeated measurements. Repeated measurements of social media use may have made employees more acutely conscious of their behaviour, resulting in a systematic decline in social media use over the course of the working day. Our results, however, do not corroborate this concern, because they showed a u-shaped trend in personal social media use throughout the working day with greater use at the beginning and towards the end of the working day. It is thus unlikely that employees restricted their personal social media use due to the repeated hourly assessments.

It should also be noted that our sample was a convenience sample and that participants were relatively young (33.8 years) and not representative for the entire working population. Recent findings on the convenience sampling method suggest that the external validity of this method is better than previously assumed (e.g., Demerouti \& Rispens, 2014; Wheeler, Shanine, Leon, \& Whitman, 2014). It is likely that younger employees were more interested in the topic of our study (social media use), because it may be more personally relevant to them than to older employees. One might argue that younger employees may be the most interesting target group when studying the phenomenon of social media use at work because they use it more frequently and for them, using social media is a matter of course.

Finally, the effects found were rather small and social media use explained only a fraction of variance in creativity and work-nonwork balance. As participants devoted only a small share of their total hourly working time (i.e., 4.5 minutes) to social media use, this 
result is not surprising. Nevertheless, the results of our study shed light on the phenomenon of social media use at work, showing that this relatively new, but common behavior explains a significant — albeit small—share of variance in important work-related outcomes (worknonwork balance and creativity).

\section{Practical Implications}

The findings of this study show that personal social media use is a common behaviour at the workplace. In our sample, personal social media was used for 4.54 minutes per hour, which amounts to about 41 minutes per working day. It also appeared that employees use social media more at the beginning and towards the end of the working day. This suggests that social media use may facilitate the transition between different spheres of life (i.e., reattachment to work and detachment from work, Sonnentag \& Kühnel, 2016).

We found that personal social media use was related to better work-nonwork balance. Thus, allowing employees to use social media reduces work-nonwork imbalance, which is related to important work-related outcomes (e.g., Amstad et al, 2011; Niedhammer, Chastang, Sultan-Taïeb, Vermeylen, \& Parent-Thirion, 2012). However, our results also showed that personal social media use was negatively related to creativity. Thus, allowing employees to use social media at work for personal purposes may have negative effects in terms of job performance. In terms of practical implications, we suggest that measures taken to prevent social media use at work—-such as monitoring or restricting access to social media - may not only prevent undesirable concomitants of social media use, but may also signal a lack of trust, which could result in impaired work engagement and well-being (see also Coker, 2011; Martin, Wellen, \& Grimmer, 2016; Moqbel, Nevo, \& Kock, 2013). However, as the results of our study suggest that a positive relationship to work-nonwork 
balance and a slightly negative relationship to creativity coexist, there remains a definite need for future studies to arrive at evidence based best practices.

\section{Conclusion}

Using ambulatory momentary assessments across an entire working day in a large sample of employees, we found that personal social media use was related to better work-nonwork balance, but also to lower creativity. The results of our study therefore showed that personal social media use at work can have both a negative (e.g. creativity) and a positive (e.g. worknonwork balance) relationship to important work-related outcomes. With this study we challenged the rather one-sided view of personal social media use as workplace delinquency. More research is needed to further elucidate the relationships between the personal use of social media during the working day, balancing work and private life, and employees' wellbeing and performance. 


\section{References}

Allen, T. D., Herst, D. E., Bruck, C. S., \& Sutton, M. (2000). Consequences associated with work-to-family conflict: a review and agenda for future research. Journal of Occupational Health Psychology, 5, 278-308. doi:10.1037/1076-8998.5.2.278

Amabile, T. M., Barsade, S. G., Mueller, J. S., \& Staw, B. M. (2005). Affect and creativity at work. Administrative Science Quarterly, 50, 367-403. doi:10.2189/asqu.2005.50.3.367

Amstad, F. T., Meier, L. L., Fasel, U., Elfering, A., \& Semmer, N. K. (2011). A metaanalysis of work-family conflict and various outcomes with a special emphasis on cross-domain versus matching-domain relations. Journal of Occupational Health Psychology, 16, 151-69. doi:10.1037/a0022170

Andreassen, C. S., Torsheim, T., \& Pallesen, S. (2014a). Use of online social network sites for personal purposes at work: Does it impair self-reported performance? Comprehensive Psychology, 3, 1-21. doi:10.2466/01.21.CP.3.18

Andreassen, C. S., Torsheim, T., \& Pallesen, S. (2014b). Predictors of use of social network sites at work-A specific type of cyberloafing. Journal of Computer-Mediated Communication, 19, 906-921. doi:10.1111/jcc4.12085

Baas, M., De Dreu, C. K. W., \& Nijstad, B. A. (2008). A meta-analysis of 25 years of moodcreativity research: Hedonic tone, activation, or regulatory focus? Psychological Bulletin, 134, 779-806. doi:10.1037/a0012815

Baethge, A., Rigotti, T., \& Roe, R. (2015). Just more of the same, or different? An integrative theoretical framework for the study of cumulative interruptions at work. European Journal of Work and Organizational Psychology, 24, 308-323. doi:10.1080/1359432X.2014.897943

Beal, D. J., Weiss, H. M., Barros, E., \& MacDermid, S. M. (2005). An episodic process 
model of affective influences on performance. Journal of Applied Psychology, 90, 1054-1068. doi:10.1037/0021-9010.90.6.1054

Beal, D. J., \& Weiss, H. M. (2003). Methods of ecological momentary assessment in organizational research. Organizational Research Methods, 6, 440-464. doi:10.1177/1094428103257361

Beauregard, T. A., \& Henry, L. C. (2009). Making the link between work-life balance practices and organizational performance. Human Resource Management Review, 19, 9-22. doi:10.1016/j.hrmr.2008.09.001

Becker, T. E., Atinc, G., Breaugh, J. A., Carlson, K. D., Edwards, J. R., \& Spector, P. E. (2016). Statistical control in correlational studies: 10 essential recommendations for organizational researchers. Journal of Organizational Behavior, 37, 157-167. doi:10.1002/job.2053

Bledow, R., Rosing, K., \& Frese, M. (2013). A dynamic perspective on affect and creativity. Academy of Management Journal, 56, 432-450. doi:10.5465/amj.2010.0894

Bliese, P. D., \& Ployhart, R. E. (2002). Growth modeling using random coefficient models: Model building, testing, and illustrations. Organizational Research Methods, 5, 362387. doi:10.1177/109442802237116

Block, R. A., \& Zakay, D. (1997). Prospective and retrospective duration judgments: A metaanalytic review. Psychonomic Bulletin \& Review, 4, 184-197. doi:10.3758/BF03209393

Bolger, N., Davis, A., \& Rafaeli, E. (2003). Diary methods: capturing life as it is lived. Annual Review of Psychology, 54, 579-616. doi:10.1146/annurev.psych.54.101601.145030

Carlson, D. S., Kacmar, K. M., Grzywacz, J. G., Tepper, B., \& Whitten, D. (2013). Workfamily balance and supervisor appraised citizenship behavior: The link of positive 
affect. Journal of Behavioral and Applied Management, 14, 87-106. doi:10.1037/t03592-000

Chen, C.-J., \& Huang, J.-W. (2009). Strategic human resource practices and innovation performance — The mediating role of knowledge management capacity. Journal of Business Research, 62, 104-114. doi:10.1016/j.jbusres.2007.11.016

Clark, S. C. (2000). Work/family border theory: A new theory of work/family balance. Human Relations, 53, 747-770. doi:10.1177/001872670053600

Coker, B. L. S. (2011). Freedom to surf: The positive effects of workplace internet leisure browsing. New Technology, Work and Employment, 26, 238-247. doi:10.1111/j.1468005X.2011.00272.x

Collins, M. A., \& Amabile, T. M. (1999). Motivation and creativity. In R. J. Sternberg (Ed.), Handbook of creativity (pp. 297-312). Cambridge: Cambridge University Press.

D'Abate, C. P. (2005). Working hard or hardly working: A study of individuals engaging in personal business on the job. Human Relations, 58, 1009-1032. doi:10.1177/0018726705058501

Demerouti, E., \& Rispens, S. (2014). Improving the image of student-recruited samples: A commentary. Journal of Occupational and Organizational Psychology, 87, 34-41. doi:10.1111/joop. 12048

Dijksterhuis, A., \& Meurs, T. (2006). Where creativity resides: The generative power of unconscious thought. Consciousness and Cognition, 15, 135-146. doi:10.1016/j.concog.2005.04.007

Dormann, C., \& Griffin, M. A. (2015). Optimal time lags in panel studies. Psychological Methods, 20, 489-505. doi:10.1037/met0000041

Duggan, M., Ellison, N. B., Lampe, C., Lenhart, A., \& Madden, M. (2015). Social media update 2014. Retrieved 9 January 2015, from Pew Research Center: 
http://www.pewinternet.org/2015/01/09/social-media-update-2014/

Edwards, A. (2013). (How) do participants in online discussion forums create 'echo chambers'? The inclusion and exclusion of dissenting voices in an online forum about climate change. Journal of Argumentation in Context, 2, 127-150. doi:10.1075/jaic.2.1.06edw

Fredrickson, B. L. (2001). The role of positive emotions in positive psychology: The broaden-and-build theory of positive emotions. American Psychologist, 56, 218-226. doi:10.1037/0003-066X.56.3.218

Friedman, W. H. (2001). Virtual absenteeism. In J. D. Haynes (Ed.), Internet management issues: A global perspective (pp. 138-149). Hershey, PA: Idea Group Publishing.

George, J. M., \& Zhou, J. (2001). When openness to experience and conscientiousness are related to creative behavior: An interactional approach. Journal of Applied Psychology, 86, 513-524. doi:10.1037/0021-9010.86.3.513

Geurts, S. A. E., Taris, T. W., Kompier, M. A. J., Dikkers, J. S. E., Van Hooff, M. L. M. \& Kinnunen, U. M. (2005). Work-home interaction from a work psychological perspective: Development and validation of a new questionnaire, the SWING. Work \& Stress, 19, 319-339. doi:10.1080/02678370500410208

Guilford, J. P. (1979). Some incubated thoughts on incubation. Journal of Creative Behavior, 13, 1-8. doi:10.1002/j.2162-6057.1979.tb00184.x

Harari, M. B., Reaves, A. C., \& Viswesvaran, C. (2016). Creative and innovative performance: A meta-analysis of relationships with task, citizenship, and counterproductive job performance dimensions. European Journal of Work and Organizational Psychology, 25, 495-511. doi:10.1080/1359432X.2015.1134491

Henle, C. A., Kohut, G., \& Booth, R. (2009). Designing electronic use policies to enhance employee perceptions of fairness and to reduce cyberloafing: An empirical test of 
justice theory. Computers in Human Behavior, 25, 902-910.

doi:10.1016/j.chb.2009.03.005

Higgins, C., Duxbury, L., \& Johnson, K. L. (2000). Part-time work for women: Does it really help balance work and family? Human Resource Management, 39, 17-32. doi:10.1002/(SICI)1099-050X(200021)39:1<17::AID-HRM3>3.0.CO;2-Y

Holland, P., \& Bardoel, A. (2016). The impact of technology on work in the twenty-first century: exploring the smart and dark side. The International Journal of Human Resource Management, 27, 2579-2581. doi:10.1080/09585192.2016.1238126

Ivarsson, L., \& Larsson, P. (2011). Personal internet usage at work: a source of recovery. Journal of Workplace Rights, 16, 63-81. doi:10.2190/WR.16.1.e

Junco, R. (2013). Comparing actual and self-reported measures of Facebook use. Computers in Human Behavior, 29, 626-631. doi:10.1016/j.chb.2012.11.007

Kaplan, A. M., \& Haenlein, M. (2010). Users of the world, unite! The challenges and opportunities of Social Media. Business Horizons, 53, 59-68.

doi:10.1016/j.bushor.2009.09.003

Kelloway, E. K., \& Barling, J. (2000). Knowledge work as organizational behavior. International Journal of Management Reviews, 2, 287-304. doi:10.1111/14682370.00042

König, C. J., \& Caner de la Guardia, M. E. (2014). Exploring the positive side of personal internet use at work: Does it help in managing the border between work and nonwork? Computers in Human Behavior, 30, 355-360. doi:10.1016/j.chb.2013.09.021

Kossek, E. E., \& Ozeki, C. (1998). Work-family conflict, policies, and the job-life satisfaction relationship: A review and directions for organizational behavior-human resources research. Journal of Applied Psychology, 83, 139-149. doi:10.1037/0021- 
9010.83 .2 .139

Kubicek, B., \& Tement, S. (2016). Work intensification and the work-home interface. Journal of Personnel Psychology, 5, 76-89. doi:10.1027/1866-5888/a000158.

Kühn, S., Ritter, S. M., Müller, B. C. N., van Baaren, R. B., Brass, M., \& Dijksterhuis, A. (2013). The importance of the default mode network in creativity-A structural MRI study. The Journal of Creative Behavior, 48, 152-163. doi:10.1002/jocb.45

Lavoie, J. A. A., \& Pychyl, T. A. (2001). Cyberslacking and the procrastination superhighway a web-based survey of online procrastination, attitudes, and emotion. Social Science Computer Review, 19, 431-444. doi:10.1177/089443930101900403

Lim, V. K. G. (2002). The IT way of loafing on the job: cyberloafing, neutralizing and organizational justice. Journal of Organizational Behavior, 23, 675-694. doi:10.1002/job.161

Lim, V. K. G., \& Chen, D. J. Q. (2012). Cyberloafing at the workplace: Gain or drain on work? Behaviour \& Information Technology, 31, 343-353. doi:10.1080/01449290903353054

Lin, L. H. (2011). Electronic human resource management and organizational innovation: the roles of information technology and virtual organizational structure. The International Journal of Human Resource Management, 22, 235-257. doi:10.1080/09585192.2011.540149

Lubart, T. I. (2001). Models of the creative process: Past, present and future. Creativity Research Journal, 13, 295-308. doi:10.1207/S15326934CRJ1334_07

Lundgren, T. D., \& Lundgren, C. A. (1999). Computer gold-bricking. The Journal of Computer Information Systems, 39, 58-62.

Maertz Jr, C. P., \& Boyar, S. L. (2011). Work-family conflict, enrichment, and balance under "levels" and "episodes" approaches. Journal of Management, 37, 68-98. 
doi:10.1177/0149206310382455

McCrae, R. R., Arenberg, D., \& Costa, P. T. (1987). Declines in divergent thinking with age: Cross-sectional, longitudinal, and cross-sequential analyses. Psychology and Aging, 2, $130-137$.

Major, D. A., \& Germano, L. M. (2006). The changing nature of work and its impact on the work-home interface. In F. Jones, R. J. Burke, \& M. Westman (Eds.), Work-life balance: A psychological perspective (pp. 13-38). New York, NY: Psychology Press.

Martin, A. J., Wellen, J. M., \& Grimmer, M. R. (2016). An eye on your work: How empowerment affects the relationship between electronic surveillance and counterproductive work behaviours. The International Journal of Human Resource Management, 27, 2635-2651. doi:10.1080/09585192.2016.1225313

Matthews, W. J., \& Meck, W. H. (2014). Time perception: the bad news and the good. Wiley Interdisciplinary Reviews: Cognitive Science, 5, 429-446. doi:10.1002/wcs.1298

Maxwell, S. E., Cole, A. D., \& Mitchel, M. A. (2011). Bias in cross-sectional analyses of longitudinal mediation. Partial and complete mediation under an autoregressive model. Multivariate Behavioral Research, 46, 816-841. doi:10.1080/00273171.2011.606716

Moqbel, M., Nevo, S., \& Kock, N. (2013). Organizational members' use of social networking sites and job performance: An exploratory study. Information Technology \& People, 26(3), 240-264. doi:10.1108/ITP-10-2012-0110

Newell, S., Robertson, M., Scarbrough, H., \& Swan, J. (2009). Managing knowledge work and innovation (Vol. 2). Houndmills: Palgrave Macmillan.

Niedhammer, I., Chastang, J. F., Sultan-Taïeb, H., Vermeylen, G., \& Parent-Thirion, A. (2012). Psychosocial work factors and sickness absence in 31 countries in Europe. The European Journal of Public Health, 23, 622-629. doi:10.1093/eurpub/cks124 
Ohly, S., Sonnentag, S., Niessen, C., \& Zapf, D. (2010). Diary studies in organizational research. Journal of Personnel Psychology, 9, 79-93. doi:10.1027/1866-5888/a000009

Olson-Buchanan, J. B., Boswell, W. R., \& Morgan, T. J. (2016). The role of managing technology in managing the work and nonwork interface. In T. D. Allen \& L. T. Eby (Eds.), Oxford handbook of work and family (pp. 333-348). New York, NY: Oxford Press.

Perrin, A. (2015). Social Media Usage: 2005-2015. Retrieved 30th November 2015, from Pew Research Center http://www.pewinternet.org/2015/10/08/social-networkingusage-2005-2015/

Pinheiro, J. C., \& Bates, D. M. (2000). Mixed effects models in S and S-PLUS. Berlin: Springer.

Raudenbush, S. W., \& Bryk, A. S. (2002). Hierarchical linear models: Applications and data analysis methods (2nd ed.). Newbury Park, CA: Sage.

Reis, H. T. (2012). Why researchers should think "real-world": A conceptual rationale. In M. R. Mehl \& T. S. Conner, Handbook of research methods for studying daily life (pp. 321). New York: Guilford Press.

Ritter, S. M. (2012). Creativity: understanding and enhancing creative thinking. (PhD), Radboud University Nijmegen, Nijmegen (Netherlands). Retrieved from http://hdl.handle.net/2066/102403

Robinson, S. L., \& Bennett, R. J. (1995). A typology of deviant workplace behaviors: A multidimensional scaling study. Academy of Management Journal, 38, 555-572. doi:10.2307/256693

Runco, M. A. (2004). Creativity. Annual Review of Psychology, 55, 657-687. doi:10.1146/annurev.psych.55.090902.141502

Ryan, R. M., \& Deci, E. L. (2000). Self-determination theory and the facilitation of intrinsic 
motivation, social development, and well-being. American Psychologist, 55, 68-78. doi:10.1037/0003-066X.55.1.68

Ryan, R. M., Bernstein, J. H., \& Brown, K. W. (2010). Weekends, work, and well-being: Psychological need satisfactions and day of the week effects on mood, vitality, and physical symptoms. Journal of Social and Clinical Psychology, 29, 95-122. doi:10.1521/jscp.2010.29.1.95

Saxbe, D. E., Repetti, R. L., \& Graesch, A. P. (2011). Time spent in housework and leisure: Links with parents' physiological recovery from work. Journal of Family Psychology, 25, 271-281. doi:10.1037/a0023048

Schooler, J. W., \& Melcher, J. (1995). The ineffability of insight. In S. M. Smith, T. B. Ward \& R. A. Finke (Eds.), The creative cognition approach (pp. 97-133). Cambridge, MA: The MIT Press.

Scott, G. M., Leritz, L. E., \& Mumford, M. D. (2004). The effectiveness of creativity training: A quantitative review. Creativity Research Journal, 16, 361-388. doi:10.1207/s15326934crj1604_1

Semmer, N. K., Zapf, D., \& Dunckel, H. (1999). Instrument zur Stressbezogenen Tätigkeitsanalyse ISTA [Instrument for stress-related job analysis]. In H. Dunckel (Ed.), Handbuch psychologischer Arbeitsanalyseverfahren (pp. 179-204). Zürich: vdf Hochschulverlag.

Simonton, D. K. (1997). Creative productivity: A predictive and explanatory model of career trajectories and landmarks. Psychological Review, 104, 66-89. doi:10.1037/0033295X.104.1.66

Simonton, D. K. (1999). Creativity as blind variation and selective retention: Is the creative process Darwinian? Psychological Inquiry, 10, 309-328.

Simonton, D. K. (2010). Creative thought as blind-variation and selective-retention: 
Combinatorial models of exceptional creativity. Physics of Life Reviews, 7, 156-179. doi:10.1016/j.plrev.2010.02.002

Sio, U. N., \& Ormerod, T. C. (2009). Does incubation enhance problem solving? A metaanalytic review. Psychological Bulletin, 135, 94-120. doi:10.1037/a0014212

Sio, U. N., Monaghan, P., \& Ormerod, T. (2013). Sleep on it, but only if it is difficult: Effects of sleep on problem solving. Memory \& Cognition, 41, 159-166. doi:10.3758/s13421012-0256-7

Snijders, T. A., \& Bosker, R. J. (1994). Modeled variance in two-level models. Sociological Methods \& Research, 22, 342-363. doi:10.1177/0049124194022003004

Sonnentag, S., \& Kühnel, J. (2016). Coming back to work in the morning: Psychological detachment and reattachment as predictors of work engagement. Journal of Occupational Health Psychology, 41, 378-390. doi:10.1037/ocp0000020

Sonnentag, S., \& Pundt, A. (2017). Media use and well-being at the work-home interface. In L. Reinecke \& M. B. Oliver (Eds.), The Routledge handbook of media use and wellbeing (pp. 341-354). Routledge: New York, NY.

Sparks, K., Faragher, B., \& Cooper, C. L. (2001). Well-being and occupational health in the 21st century workplace. Journal of Occupational and Organizational Psychology, 74, 489-509. doi:10.1348/096317901167497

Spector, P. E., \& Brannick, M. T. (2011). Methodological urban legends: The misuse of statistical control variables. Organizational Research Methods, 14, 287-305. doi:10.1177/1094428110369842

Syrek, C. J., \& Antoni, C. H. (2014). Unfinished tasks foster rumination and impair sleeping-Particularly if leaders have high performance expectations. Journal of Occupational Health Psychology, 19, 490-499. doi:10.1037/a0037127

Tan, T. F., \& Netessine, S. (2014). When does the devil make work? An empirical study of 
the impact of workload on worker productivity. Management Science, 60, 1574-1593. doi:10.1287/mnsc. 2014.1950

Tingley, D., Yamamoto, T., Hirose, K., Keele, L., \& Imai, K. (2014). Mediation: R package for causal mediation analysis. Journal of Statistical Software, 59, 1-38. doi:10.18637/jss.v059.i05

Vitak, J., Crouse, J., \& LaRose, R. (2011). Personal Internet use at work: Understanding cyberslacking. Computers in Human Behavior, 27, 1751-1759. doi:10.1016/j.chb.2011.03.002

Weatherbee, T. G. (2010). Counterproductive use of technology at work: Information and communications technologies and cyberdeviancy. Human Resource Management Review, 20, 35-44. doi:10.1016/j.hrmr.2009.03.012

Wheeler, A. R., Shanine, K. K., Leon, M. R., \&Whitman, M. V. (2014). Student-recruited samples in organizational research: A review, analysis, and guidelines for future research. Journal of Occupational and Organizational Psychology, 87, 1-26. doi:10.1111/joop.12042

Whetten, D. A., \& Cameron, K. S. (1998). Developing management skills (4th ed.). Reading, MA: Addison Wesley Longman. 


\section{Footnote}

${ }^{1}$ Time was negatively linearly and positively quadratically related to work-nonwork balance and a random slope for the linear trend improved model fit $\left(\Delta \chi^{2}=15.60, d f=2, p<.001\right)$. Model fit was best for a model including autocorrelation. Heterogeneity in the error structures was not relevant. The basic model for work-nonwork balance (Model 1, Table 2) included a random intercept and a random slope for the linear time trend $(\beta=-3.77, S E=1.68, t=-2.25$, $p<.05)$, a random intercept for the quadratic time trend $(\beta=6.53, S E=1.57, t=4.15, p<$ .001 ), and autocorrelation. For creativity, neither the linear nor the quadratic time trend was a significant predictor. A model (Model 1, Table 3) including a random slope for the linear time trend $(\beta=-.002, S E=.01, t=-0.20, p=.84)$ improved model fit $\left(\Delta \chi^{2}=37.31, d f=2, p\right.$ $<.001$ ), indicating that the linear trend over time differed between employees. Model fit was best for a model including autocorrelation, while heterogeneity in the error structures was not relevant. 
Table 1. Means, standard deviations and correlations between study variables

\begin{tabular}{|c|c|c|c|c|c|c|c|c|c|c|}
\hline Variable & $M$ & $S D$ & 1 & 2 & 3 & 4 & 5 & 6 & 7 & 8 \\
\hline 1 Personal social media use & 4.54 & 6.79 & & $-.06 * *$ & $.35 * * *$ & $-.11 * * *$ & & & & \\
\hline 2 Creativity & 3.80 & 1.47 & $-.17 * *$ & & -.04 & -.01 & & & & \\
\hline 3 Work-nonwork balance & 3.33 & 1.88 & $.19 * * *$ & .00 & & $-.09 * * *$ & & & & \\
\hline 4 Initiator of contact & 4.45 & 2.56 & .02 & .01 & $-.11 *$ & & & & & \\
\hline 5 Gender & 1.50 & .49 & $-.12 *$ & -.04 & $.14^{*}$ & .06 & & & & \\
\hline 6 Age & 33.80 & 10.37 & $-.19 * * *$ & $.15^{* *}$ & $.13 *$ & -.10 & $-.15 * *$ & & & \\
\hline 7 Family status & .62 & .47 & $-.20 * * *$ & .08 & .09 & -.03 & -.02 & $.46^{* * *}$ & & \\
\hline 8 Children & 1.76 & .42 & .14 & .03 & -.07 & .10 & $.14^{*}$ & $.50 * * *$ & $-.35 * * *$ & \\
\hline 9 Job autonomy & 3.61 & .76 & $-.13 *$ & $.14^{* *}$ & .08 & -.06 & $-.15^{* *}$ & $.13 *$ & $.14 *$ & -.07 \\
\hline
\end{tabular}

Note. Correlations below the diagonal are person-level correlations $(N=337 ; 332$ for creativity), correlations above the diagonal are hour-level correlations between person-mean centred variables $(N=2244 ; 2224$ for creativity $)$ Gender: $1=$ male, $2=$ female, Family status: $0=$ single, $1=$ in a relationship, Children living at home: $1=y e s, 2=n o .{ }^{* * *} p<.001,{ }^{* *} p<.01,{ }^{*} p<.05$. 
Table 2. Multilevel regression analyses predicting work-nonwork balance

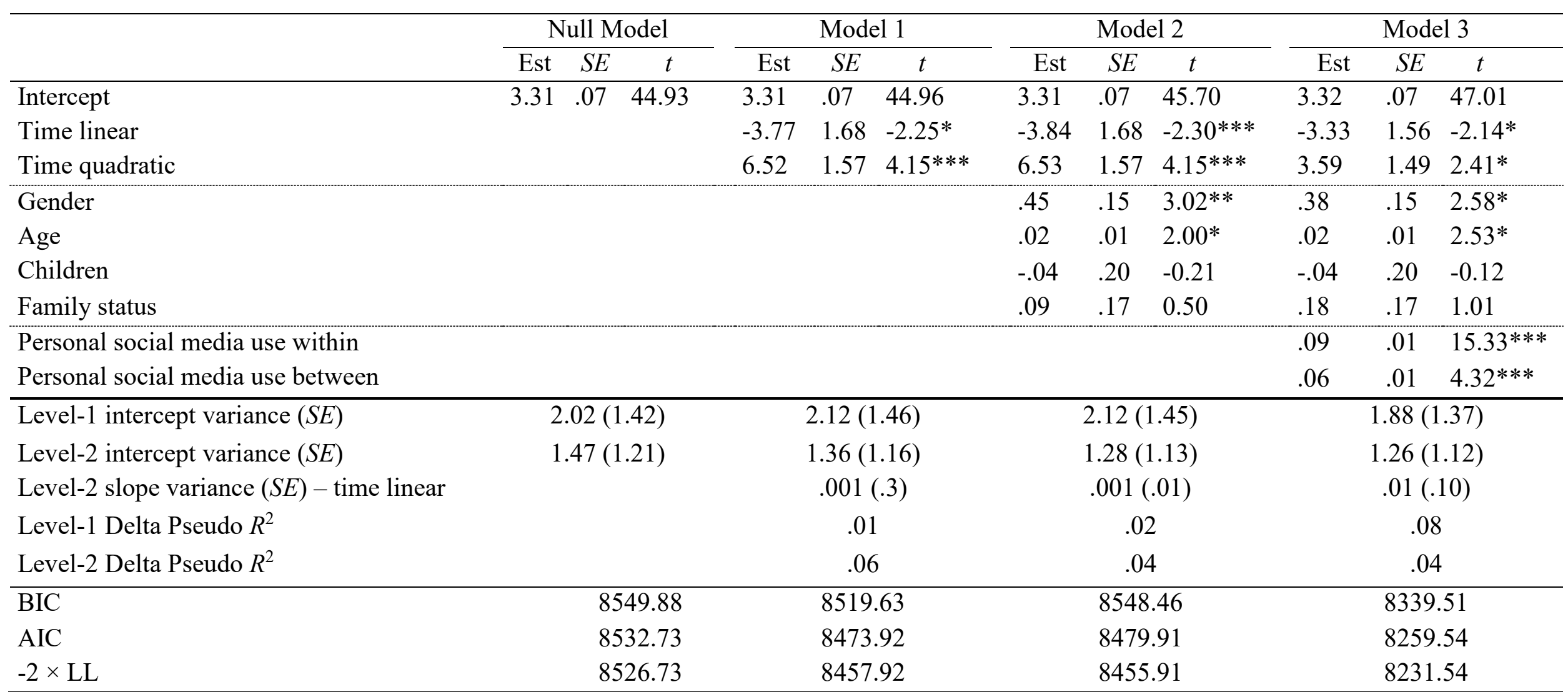

Note. Est $=$ Estimate. $N_{\text {Level } 2}=337, N_{\text {Level1 }}=2244$. For the calculation of Delta Pseudo $R^{2}$, we used the formulas of Snijders and Bosker $(1994)$.

For the calculation of Delta Pseudo $R^{2}$, models without the random slope for time were estimated and compared. $* * * p<.001, * * p<.01, * p<$ .05 . 
Table 3. Multilevel regression analyses predicting creativity

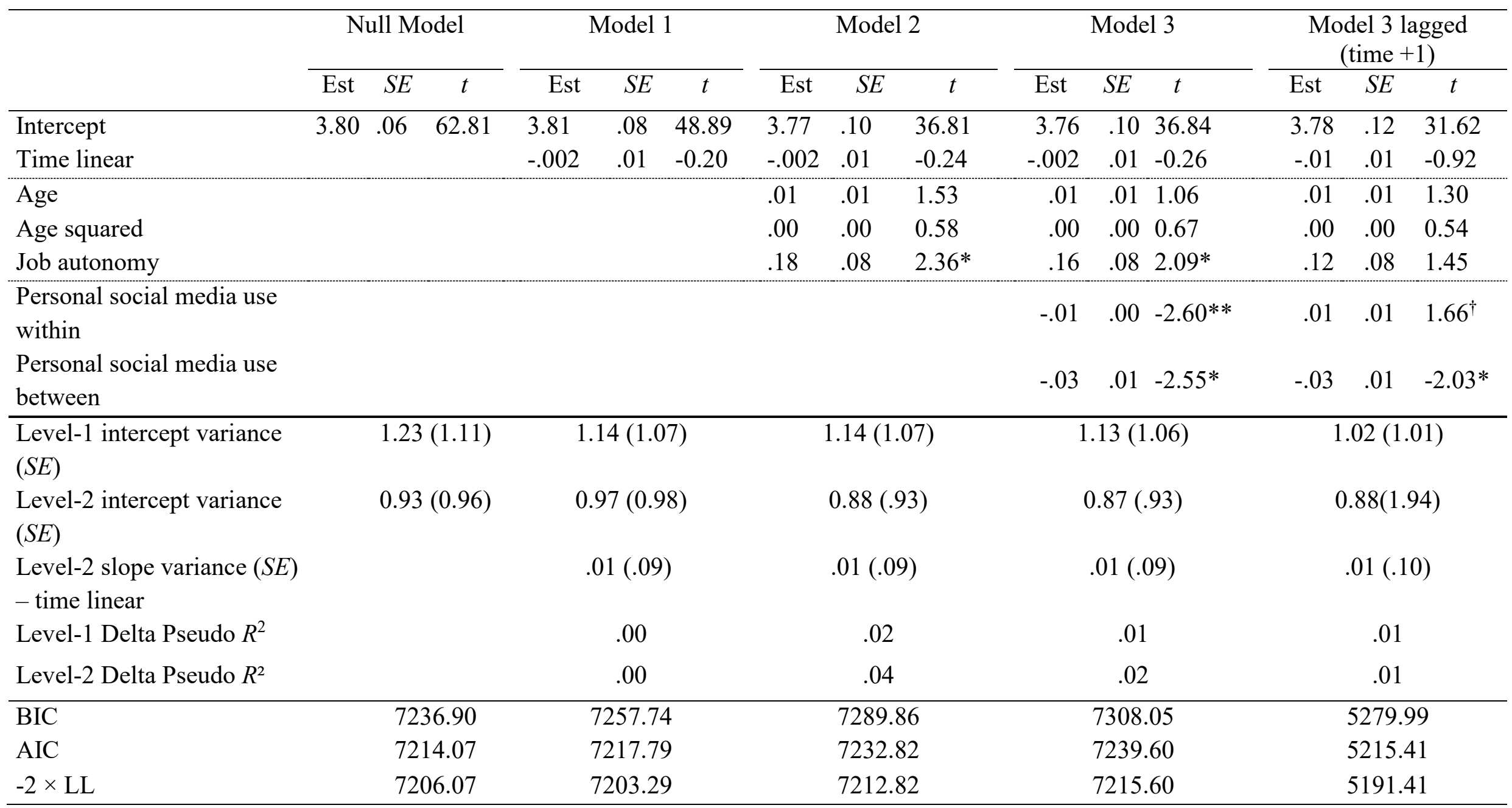

Note. Est $=$ Estimate. Null Model to Model 3: $N_{\text {Level2 }}=332, N_{\text {Level1 }}=2224$; Model 3 lagged: $N_{\text {Level2 }}=312, N_{\text {Level1 }}=1613$. Model 3 lagged is 
(Model 2 lagged is not depicted in Table 3; fit indices were $\mathrm{BIC}=5255.37, \mathrm{AIC}=5212.30,-2 \times \mathrm{LL}=5196.30$ ). For the calculation of Delta Pseudo $R^{2}$ we used the formulas of Snijders and Bosker (1994). For the calculation of Delta Pseudo $R^{2}$, models without the random slope for time were estimated and compared. $* * * p<.001, * * p<.01, * p<.05,{ }^{\dagger} p<.10$. 
Figure 1. Conceptual model of hypothesized effects.

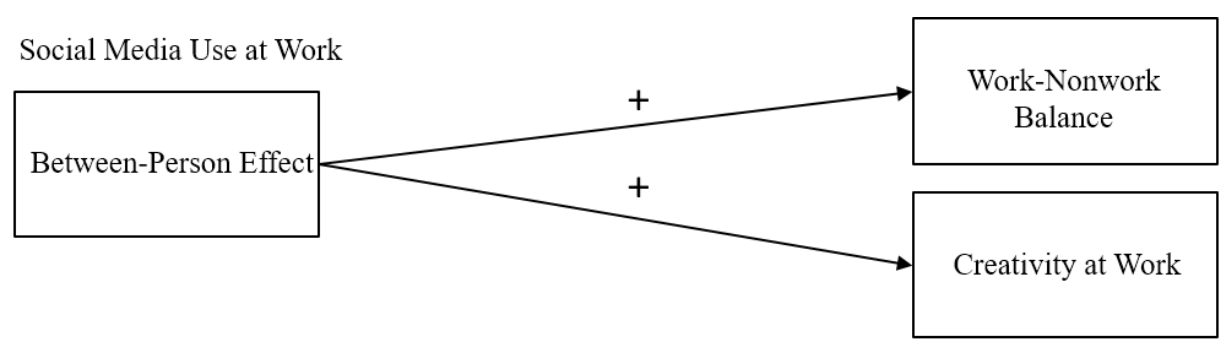

Day-Level of Analysis (between person)



Hour-Level of Analysis (within person) 